OPEN ACCESS

Edited by:

Fushun Wang,

Nanjing University of Chinese

Medicine, China

Reviewed by:

Bing Xiang Yang,

Wuhan University, China

Abhilash Perisetti,

University of Arkansas for Medical

Sciences, United States

*Correspondence:

Jingping Zhang

jpzhang1965@csu.edu.cn

Specialty section

This article was submitted to

Psychology for Clinical Settings,

a section of the journal

Frontiers in Psychology

Received: 10 October 2020 Accepted: 07 May 2021

Published: 07 June 2021

Citation:

Li Y, Li J, Yang Z, Zhang J, Dong L, Wang F and Zhang J (2021)

Gender Differences in Anxiety,

Depression, and Nursing Needs Among Isolated Coronavirus Disease 2019 Patients.

Front. Psychol. 12:615909 doi: 10.3389/fpsyg.2021.615909

\section{Gender Differences in Anxiety, Depression, and Nursing Needs Among Isolated Coronavirus Disease 2019 Patients}

\author{
Yifei Li ${ }^{1}$, Juan $\mathrm{Li}^{1}$, Zhen Yang ${ }^{2}$, Jie Zhang ${ }^{1}$, Lili Dong ${ }^{2}$, Fusheng Wang ${ }^{2}$ and \\ Jingping Zhang ${ }^{1 *}$ \\ ${ }^{1}$ Nursing Psychology Research Center, Xiangya School of Nursing, Central South University, Changsha, China, ${ }^{2}$ The \\ Second Affiliated Hospital of Shantou University Medical College, Shantou, China
}

Objective: This study explored gender differences in anxiety, depression, and nursing needs among isolated Coronavirus Disease 2019 (COVID-19) patients, with a particular focus on the influencing factors. The main goal was to elucidate breakthrough points and intervention targets for psychological counseling aimed at the promotion of overall health during isolation treatment.

Methods: A survey was conducted to obtain information about the nursing needs of COVID-19 patients, with mental health assessed via the Hospital Anxiety and Depression Scale (HADS). Participants included 219 isolated COVID-19 patients at a Wuhan module hospital in Hubei province, China.

Results: A total of 216 valid questionnaires were collected (98.63\% retrieval rate). Of these participants, $21.76 \%$ had anxiety symptoms, while $17.59 \%$ had depression symptoms. Colleagues infected with COVID-19 (OR $=3.896,95 \% \mathrm{Cl}: 1.555-9.764$, $P=0.004$ ) were the main influencing factors for anxiety symptoms, while marital status $(\mathrm{OR}=2.700,95 \% \mathrm{Cl}: 1.033-7.055, P=0.043)$ and family members infected with COVID-19 (OR $=2.969,95 \% \mathrm{Cl}: 1.243-7.095, P=0.014)$ were the main influencing factors for depression symptoms. As for gender, male patients were generally more prone to depression and anxiety than female patients, especially those who were infected with colleagues. On the other hand, female patients reported greater concerns about safe treatment environments and communication with medical staff.

Conclusion: This study found gender-based differences regarding the factors influencing anxiety and depression in isolated COVID-19 patients, with males reporting a greater general tendency for symptoms. On the other hand, female patients reported greater overall psychological nursing needs than males. Targeted nursing should thus be implemented to address specific psychological characteristics and nursing needs.

Keywords: coronavirus disease 2019, isolated patients, gender, nursing needs, hospital anxiety and depression scale, COVID-19 


\section{INTRODUCTION}

The highly contagious acute respiratory infection leading to the illness known as Coronavirus Disease 2019 (COVID-19) began spreading across the globe in late 2019 (Wang C. et al., 2020). It has now infected more than 149 million people, resulting in 3.15 million total deaths as of April 30, 2021 (World Health Organization (WHO), 2021). COVID-19 is thus referred to as the sixth worldwide public health crisis (Arab-Mazar et al., 2020). According to the existing case data, COVID-19 is mainly manifested by fever, dry cough and fatigue, and a small number of patients are accompanied by upper respiratory tract and digestive tract symptoms such as nasal obstruction, runny nose and diarrhea. About half of the patients develop dyspnea 1 week later, which in severe cases can lead to acute respiratory distress syndrome or septic shock and even death (Chen and Li, 2020). The primary routes of transmission of the COVID-19 are through respiratory droplets and close person-to-person contact (Amirian, 2020).

In this context, quarantine is one of the most effective measures for halting the spread of the virus (Chen Z. et al., 2020). In fact, the diagnosis and treatment plan issued by the National Health Commission of the People's Republic of China also emphasizes that COVID-19 patients must be isolated and treated in designated hospitals (National Health Commission of the People's Republic of China, 2020). However, this practice has created a dilemma. While isolation provides epidemiological benefits, it can also cause psychosocial harm, thereby resulting in more negative emotions and nursing needs among affected patients (Sharma et al., 2020).

A previous study on severe acute respiratory syndrome (SARS) patients who were placed in isolation found that 96.6\% thus experienced feelings of inferiority, loneliness, and abandonment (Yang, 2004). A later follow-up study on discharged SARS patients found that more than one-third had incurred psychological problems, including post-traumatic stress disorder, anxiety, and depression (Kwek et al., 2006). Similarly, an investigation among isolated COVID-19 patients found that anxiety and depression were common psychological problems, with respective incidence rates of 38.5 and $35.9 \%$ (Nie et al., 2020). These negative psychological impacts not only harm mental health, but may also affect disease recovery (Fu and Luo, 2011; Pang, 2016).

One of the important purposes of human activity is to satisfy needs. In this regard, isolated COVID-19 patients may require greater nursing needs due to several unique conditions, including separation from relatives, fear of the disease, and unfamiliarity induced by isolation (Fan et al., 2020). Wang Z. Y. et al. (2020) found that COVID-19 patients generally experienced anxiety and depression when being admitted to the hospital. However, most of these symptoms are alleviated through continued treatment and satisfaction with nursing services (Wang Z. Y. et al., 2020). In other words, medical staff can more efficiently deal with negative emotions through a better understanding of the specific nursing needs, thus improving overall mental health for patients.

Many previous studies on COVID-19 patients have found gender-based differences in both anxiety and depression. For example, female patients are more likely to experience negative emotions related to anxiety and depression than male patients (Gu et al., 2020; Nie et al., 2020). Similar studies on SARS patients have also found that males and females tend to have different nursing needs, with female patients reporting a greater need for contact with relatives ( $\mathrm{Li}$ et al., 2003). These types of studies can produce valuable information for medical staff during targeted interventions aimed at reducing negative emotions for patients. However, there is still a lack of information on gender-based differences in anxiety, depression, and nursing needs among COVID-19 patients. As such, this survey study investigated differences in sociodemographic characteristics, anxiety, depression, and nursing needs between male and female COVID-19 patients who were placed in isolation. Our findings provide a basis for interventions and services aimed at alleviating negative emotions while promoting overall mental health.

\section{MATERIALS AND METHODS}

This study implemented a cross-sectional design in which an online survey was conducted to assess anxiety, depression, and nursing needs among isolated COVID-19 patients at a Wuhan module hospital in Hubei province, China. All participants admitted to the hospital were symptomatic patients with mild COVID-19. The data collection period lasted from March 3$7,2020$.

\section{Participants}

Patients with COVID-19 were invited to participate in the online survey through the Wenjuanxing platform. All questionnaires were completed anonymously. Specific inclusion criteria were set as follows: patients who (1) were diagnosed with COVID19 according to treatment protocols, (2) allowed to voluntarily participate in this study, (3) provided their informed written consent. Exclusion criteria were set as follows: patients with self-reported histories of neurological disorders, mental illness, and/or other serious systemic disorders. This resulted in a total of 219 eligible participants patients. After removing data from those with incomplete questionnaires, 216 were ultimately included in the analyses. This study was reviewed by the Institutional Review Board at the researchers' university (Ethical Grant Number: E202073). All patients provided online informed consent prior to their participation.

\section{Measurements}

\section{Demographic Characteristics}

Demographic data were collected using a self-designed questionnaire, with reported characteristics including gender, age, marital status, education level, occupation, living area, monthly family income, hospitalization time, and whether any family members or friends were infected with COVID-19. Other questions included the following: "Do you have any symptoms such as fever, cough, sore throat, chest tightness, diarrhea or fatigue?" and "Have you felt any improvement since admission?". 


\section{Hospital Anxiety and Depression Scale}

Participants also completed the Hospital Anxiety and Depression Scale (HADS) (Zigmond and Snaith, 1983), which was used to determine the presence of depression and anxiety. The HADS consists of 14 items, including seven for depression and seven for anxiety. Scores for each subscale may range from $0-21$, with scores of 0-7 denoting no symptoms, 8-10 denoting borderline abnormal cases, and 11-21 denoting abnormal cases, respectively. Higher scores on each subscale reflect more severe symptoms. In this study, Cronbach's alpha values of 0.842 and 0.850 were achieved for the anxiety and depression subscales, respectively.

\section{Questionnaire on the Nursing Needs of COVID-19 Patients}

Referring to the evaluation model of "functional health patterns" established by Gordon (Sheng et al., 2003) and based on expert advice, we reviewed relevant literature (Zhang, 2012) and designed the "Questionnaire on the nursing Needs of COVID-19 patients" (Table 4). The scale consists of 18 items that are each answered with one of three options, including "need," "does not matter," and "do not need." In this study, the scale achieved a Cronbach's alpha value of 0.820 .

\section{Data Analysis}

IBM SPSS Version 21.0 was used for all statistical analyses (significance threshold set at 0.05). Continuous descriptive data were expressed as means and standard deviations (SDs), whereas categorical data were expressed as frequencies and percentages via the chi-squared test. A multivariate logistic regression was conducted to investigate potential influencing factors for anxiety and depression in three groups, including all participants, male participants, and female participants. The associations between anxiety, depression, and influencing factors were presented as odds ratios (ORs) and 95\% confidence intervals (CIs).

\section{RESULTS}

\section{Analyzing Basic Participant Characteristics}

This study analyzed data from 216 isolated COVID-19 patients, including 124 males and 92 females (average age of $39.21 \pm 9.91$; range of 18-64 years). The length of hospital stay ranged from 1-38 days, with an average of $13.51 \pm 4.17$. Of all participants, $21.76 \%$ had anxiety symptoms, while $17.59 \%$ had depression symptoms. Colleagues infected with COVID-19 were associated with anxiety $(\chi 2=7.446, P=0.006)$, while family members infected with COVID-19 were associated with depression $(\chi 2=4.743, P=0.029)$. Table 1 shows the relationship between basic characteristics and the presence of depression and anxiety symptoms.

\section{Gender-Focused Multivariate Analysis of Anxiety Symptoms}

A multivariate logistic regression analysis showed that colleagues infected with COVID-19 were the main influencing factors for anxiety symptoms $(\mathrm{OR}=3.896,95 \% \mathrm{CI}: 1.555-9.764, P=0.004)$, particularly among male participants $(\mathrm{OR}=13.286,95 \% \mathrm{CI}$ : 2.902-60.832, $P=0.001)$. For female participants, on the other hand, occupation (OR $=1.393,95 \% \mathrm{CI}$ : 1.007-1.926, $P=0.045)$ and the item "Do you have any symptoms such as fever, cough, sore throat, chest tightness, diarrhea or fatigue?" ( $\mathrm{OR}=0.255$, 95\% CI: $0.068-0.959, P=0.043$ ) were the main influencing factors of anxiety symptoms (Table 2 ).

\section{Gender-Focused Multivariate Analysis of Depression Symptoms}

A multivariate logistic regression analysis showed that marital status (OR $=2.700,95 \% \mathrm{CI}: 1.033-7.055, P=0.043)$ and family members infected with COVID-19 (OR $=2.969,95 \% \mathrm{CI}: 1.243-$ $7.095, P=0.014$ ) were the main influencing factors for depression symptoms. For male participants, the main factors included age (OR $=0.859,95 \% \mathrm{CI}: 0.767-0.962, P=0.009)$, marital status $(\mathrm{OR}=30.409,95 \% \mathrm{CI}: 3.485-265.350, P=0.002)$, monthly family income $(\mathrm{OR}=0.378,95 \% \mathrm{CI}: 0.165-0.867, P=0.022)$, family members infected with COVID-19 (OR = 19.903, 95\%CI: $2.616-$ 151.430, $P=0.004)$, and colleagues infected with COVID-19 $(\mathrm{OR}=21.639,95 \% \mathrm{CI}: 2.571-182.102, P=0.005)$. However, none of the tested factors showed statistically significant influences on depression symptoms among female participants (Table 3).

\section{Gender-Based Nursing Needs}

As shown in Table 4, timely examinations and treatments, understanding of the disease prognosis, understanding the effects and side effects of therapeutic agents, and guidance for preventing recurrence were more than $95 \%$ in both male and female participants. However, female participants reported a greater general need for three items, including being cared for by family members, safe and comfortable treatment environments, and communication with doctors and nurses. These differences were statistically significant $(P<0.05)$.

\section{DISCUSSION}

\section{Analyzing Anxiety, Depression, and the Influencing Factors for Both}

As mentioned, $21.76 \%$ of participants had anxiety symptoms, while $17.59 \%$ had depression symptoms. These results may partly be related to the fact that all were considered mild patients with generally good prognoses and low mortality risks (Xiong et al., 2020). The average length of stay was $13.51 \pm 4.17$ days. In this context, familiarity with both the inpatient environment and medical staff may have reduced anxiety and depression during the isolation period (Wang $\mathrm{Z}$. Y. et al., 2020). After receiving a series of treatments, 54.63\% believed that their condition had significantly improved, which was also conducive to the reduction of negative emotions related to general health concerns.

Participants whose colleagues were also infected with COVID19 were more prone to anxiety symptoms. This may be related to COVID-19 is highly contagious. Colleague infections mean 
TABLE 1 | Demographic characteristics of patients $(N=216)$.

\begin{tabular}{|c|c|c|c|c|c|c|c|c|c|}
\hline Variables & & $\begin{array}{c}\text { Non-anxiety, } N \\
\text { (\%) }\end{array}$ & Anxiety, $N(\%)$ & $x^{2}$ & $P$ & $\begin{array}{c}\text { Non-depression, } \\
N(\%)\end{array}$ & $\begin{array}{c}\text { Depression, } N \\
(\%)\end{array}$ & $x^{2}$ & $P$ \\
\hline \multirow[t]{2}{*}{ Gender } & Male & $102(47.22)$ & $22(10.19)$ & 2.760 & 0.097 & $102(47.22)$ & $22(10.19)$ & 0.004 & 0.947 \\
\hline & Female & 67 (31.02) & $25(11.57)$ & & & 76 (35.18) & $16(7.41)$ & & \\
\hline \multirow[t]{3}{*}{ Age, year } & $18-39$ & $93(43.05)$ & $22(10.19)$ & 3.294 & 0.193 & 92 (42.59) & $23(10.65)$ & 1.954 & 0.377 \\
\hline & $40-59$ & $70(32.41)$ & $25(11.57)$ & & & $80(37.04)$ & $15(6.94)$ & & \\
\hline & $>60$ & $6(2.78)$ & 0 & & & $6(2.78)$ & 0 & & \\
\hline \multirow[t]{4}{*}{ Marital status } & Unmarried & $34(15.74)$ & $7(3.24)$ & 1.654 & 0.647 & $37(17.13)$ & $4(1.85)$ & 3.473 & 0.324 \\
\hline & Married & $129(59.72)$ & $38(17.59)$ & & & $135(62.50)$ & $32(14.82)$ & & \\
\hline & Divorce & $4(1.85)$ & $2(0.93)$ & & & $5(2.32)$ & $1(0.46)$ & & \\
\hline & Bereft of one's spouse & $2(0.93)$ & 0 & & & $1(0.46)$ & $1(0.46)$ & & \\
\hline \multirow[t]{5}{*}{ Educational level } & Primary school or below & $3(1.39)$ & 0 & 3.153 & 0.533 & $1(0.46)$ & $2(0.93)$ & 5.289 & 0.259 \\
\hline & Junior high school & $19(8.79)$ & $9(4.17)$ & & & $23(10.64)$ & $5(2.32)$ & & \\
\hline & High school & $39(18.05)$ & $9(4.17)$ & & & $41(18.98)$ & $7(3.24)$ & & \\
\hline & Junior college or bachelor & $100(46.29)$ & $26(12.04)$ & & & $104(48.15)$ & $22(10.18)$ & & \\
\hline & Master or above & $9(4.17)$ & $2(0.93)$ & & & $9(4.17)$ & $2(0.93)$ & & \\
\hline \multirow[t]{6}{*}{ Occupation } & Students & $7(3.24)$ & 0 & 7.011 & 0.220 & $6(2.78)$ & $1(0.46)$ & 4.081 & 0.538 \\
\hline & HCWs & $2(0.93)$ & 0 & & & $2(0.93)$ & 0 & & \\
\hline & $\begin{array}{l}\text { Government employees } \\
\text { or institutional employees }\end{array}$ & $82(37.96)$ & $18(8.33)$ & & & $87(40.28)$ & $13(6.02)$ & & \\
\hline & Freelancers & $28(12.96)$ & $13(6.02)$ & & & $33(15.28)$ & $8(3.70)$ & & \\
\hline & Unemployed & $8(3.70)$ & $1(0.46)$ & & & $7(3.24)$ & $2(0.93)$ & & \\
\hline & Others & $42(19.45)$ & $15(6.95)$ & & & $43(19.90)$ & $14(6.48)$ & & \\
\hline \multirow[t]{2}{*}{ Living area } & Urban & $161(74.54)$ & $46(21.30)$ & 0.625 & 0.429 & $169(78.24)$ & $38(17.59)$ & 2.005 & 0.157 \\
\hline & Rural & $8(3.70)$ & $1(0.46)$ & & & $9(4.17)$ & 0 & & \\
\hline \multirow{4}{*}{$\begin{array}{l}\text { Monthly Family } \\
\text { income, } ¥^{\mathrm{a}}\end{array}$} & $2000<$ & $6(2.78)$ & $2(0.93)$ & 4.284 & 0.232 & $6(2.78)$ & $2(0.93)$ & 0.958 & 0.811 \\
\hline & $2001-4000$ & 28 (12.96) & $14(6.48)$ & & & $33(15.27)$ & $9(4.17)$ & & \\
\hline & $4001-6000$ & 43 (19.91) & $10(4.63)$ & & & $44(20.37)$ & $9(4.17)$ & & \\
\hline & $>6000$ & $92(42.59)$ & $21(9.72)$ & & & 95 (43.98) & 18 (8.33) & & \\
\hline \multirow{4}{*}{$\begin{array}{l}\text { Hospitalization time, } \\
\text { day }\end{array}$} & $0-7$ & $14(6.48)$ & $4(1.85)$ & 1.441 & 0.696 & $16(7.40)$ & $2(0.93)$ & 1.695 & 0.638 \\
\hline & $8-14$ & 66 (30.55) & $22(10.19)$ & & & $74(34.26)$ & $14(6.48)$ & & \\
\hline & $15-21$ & 87 (40.28) & 21 (9.72) & & & 86 (39.81) & $22(10.19)$ & & \\
\hline & $>22$ & $2(0.93)$ & 0 & & & $2(0.93)$ & 0 & & \\
\hline \multirow{2}{*}{$\begin{array}{l}\text { Are your family } \\
\text { members infected } \\
\text { with COVID-19? }\end{array}$} & No & 67 (31.02) & 18 (8.33) & 0.028 & 0.867 & 76 (35.19) & $9(4.17)$ & 4.743 & $0.029 *$ \\
\hline & Yes & $102(47.22)$ & $29(13.43)$ & & & $102(47.22)$ & $29(13.42)$ & & \\
\hline \multirow{2}{*}{$\begin{array}{l}\text { Are your friends } \\
\text { infected with } \\
\text { COVID-19? }\end{array}$} & No & $154(71.30)$ & 43 (19.91) & 0.006 & 0.938 & 165 (76.39) & $32(14.81)$ & 2.811 & 0.094 \\
\hline & Yes & 15 (6.94) & $4(1.85)$ & & & $13(6.02)$ & $6(2.78)$ & & \\
\hline \multirow{2}{*}{$\begin{array}{l}\text { Are your colleagues } \\
\text { infected with } \\
\text { COVID-19? }\end{array}$} & No & 147 (68.05) & $33(15.28)$ & 7.446 & $0.006^{*}$ & 151 (69.91) & $29(13.42)$ & 1.635 & 0.201 \\
\hline & Yes & $22(10.19)$ & $14(6.48)$ & & & 27 (12.50) & $9(4.17)$ & & \\
\hline \multirow{2}{*}{$\begin{array}{l}\text { Are your neighbors } \\
\text { infected with } \\
\text { COVID-19? }\end{array}$} & No & $152(70.37)$ & $42(19.44)$ & 0.013 & 0.908 & 159 (73.61) & $35(16.20)$ & 0.264 & 0.607 \\
\hline & Yes & $17(7.87)$ & $5(2.32)$ & & & $19(8.80)$ & $3(1.39)$ & & \\
\hline \multirow{2}{*}{$\begin{array}{l}\text { Do you have any } \\
\text { symptoms such as } \\
\text { fever, cough, sore } \\
\text { throat, chest } \\
\text { tightness, diarrhea or } \\
\text { fatigue? }\end{array}$} & No & $71(32.87)$ & $17(7.87)$ & 0.520 & 0.471 & $76(35.18)$ & $12(5.56)$ & 1.603 & 0.205 \\
\hline & Yes & 98 (45.37) & 30 (13.89) & & & $102(47.22)$ & 26 (12.04) & & \\
\hline \multirow{5}{*}{$\begin{array}{l}\text { Have you felt any } \\
\text { improvement since } \\
\text { admission? }\end{array}$} & Improved significantly & $97(44.91)$ & $21(9.72)$ & 2.536 & 0.469 & $98(45.37)$ & $20(9.26)$ & 0.281 & 0.964 \\
\hline & Slight improvement & $43(19.91)$ & $16(7.41)$ & & & 49 (22.69) & $10(4.63)$ & & \\
\hline & unchanged & $25(11.57)$ & $9(4.17)$ & & & $27(12.50)$ & $7(3.24)$ & & \\
\hline & Slight worse & $4(1.85)$ & $1(0.46)$ & & & $4(1.85)$ & $1(0.46)$ & & \\
\hline & Significantly worse & & & & & & & & \\
\hline
\end{tabular}

${ }^{a}$ As of November 18, 2020, $1 ¥=\$ 0.15$ US. ${ }^{*} P<0.05$ N, Number; HCWs, health care workers; COVID-19, coronavirus disease 2019. 
TABLE 2 | Gender-influencing factors of anxiety symptoms in isolated COVID-19 patients.

\begin{tabular}{|c|c|c|c|c|c|c|c|c|c|c|c|c|c|}
\hline \multicolumn{2}{|r|}{ Variables } & \multicolumn{4}{|c|}{ All $(N=216)$} & \multicolumn{4}{|c|}{ Male $(N=124)$} & \multicolumn{4}{|c|}{ Female $(N=92)$} \\
\hline & & $B$ & OR & $95 \% \mathrm{Cl}$ & $P$ & $B$ & OR & $95 \% \mathrm{Cl}$ & $P$ & $B$ & OR & $95 \% \mathrm{Cl}$ & $P$ \\
\hline \multirow[t]{14}{*}{ Anxiety } & Gender & 0.499 & 1.646 & $0.806-3.364$ & 0.171 & & & & & & & & \\
\hline & Age & 0.008 & 1.008 & $0.963-1.056$ & 0.725 & -0.016 & 0.984 & $0.916-1.057$ & 0.652 & 0.023 & 1.024 & $0.947-1.106$ & 0.558 \\
\hline & Marital status & 0.095 & 1.100 & $0.452-2.675$ & 0.834 & 0.736 & 2.088 & $0.442-9.873$ & 0.353 & -1.245 & 0.288 & $0.057-1.464$ & 0.134 \\
\hline & Educational level & 0.291 & 1.337 & $0.777-2.302$ & 0.294 & 0.087 & 1.091 & $0.479-2.486$ & 0.836 & 0.450 & 1.569 & $0.624-3.942$ & 0.338 \\
\hline & Occupation & 0.198 & 1.219 & $0.992-1.497$ & 0.060 & 0.186 & 1.205 & $0.877-1.656$ & 0.250 & 0.331 & 1.393 & $1.007-1.926$ & $0.045^{*}$ \\
\hline & Living area & -1.359 & 0.257 & $0.026-2.524$ & 0.244 & -19.778 & 0.000 & $0.000-0.000$ & 0.999 & 0.758 & 2.134 & $0.093-48.854$ & 0.635 \\
\hline & Monthly Family income & -0.390 & 0.677 & $0.430-1.067$ & 0.093 & -0.406 & 0.666 & $0.339-1.310$ & 0.239 & -0.182 & 0.833 & $0.393-1.768$ & 0.635 \\
\hline & Hospitalization time & -0.027 & 0.974 & $0.889-1.067$ & 0.567 & -0.102 & 0.903 & $0.780-1.045$ & 0.171 & 0.109 & 1.115 & $0.925-1.345$ & 0.253 \\
\hline & $\begin{array}{l}\text { Are your family members } \\
\text { infected with COVID-19? }\end{array}$ & 0.312 & 1.366 & $0.653-2.853$ & 0.407 & 0.963 & 2.619 & $0.740-9.269$ & 0.135 & -0.403 & 0.668 & $0.218-2.052$ & 0.481 \\
\hline & $\begin{array}{l}\text { Are your friends infected with } \\
\text { COVID-19? }\end{array}$ & -0.890 & 0.411 & $0.099-1.698$ & 0.219 & -0.390 & 0.677 & $0.075-6.079$ & 0.727 & -1.428 & 0.240 & $0.028-2.033$ & 0.190 \\
\hline & $\begin{array}{l}\text { Are your colleagues infected } \\
\text { with COVID-19? }\end{array}$ & 1.360 & 3.896 & $1.555-9.764$ & $0.004 *$ & 2.587 & 13.286 & $2.902-60.832$ & $0.001 *$ & 0.298 & 1.348 & $0.332-5.478$ & 0.677 \\
\hline & $\begin{array}{l}\text { Are your neighbors infected } \\
\text { with COVID-19? }\end{array}$ & -0.215 & 0.806 & $0.235-2.770$ & 0.732 & -1.435 & 0.238 & $0.020-2.868$ & 0.258 & 1.038 & 2.825 & $0.452-17.674$ & 0.267 \\
\hline & $\begin{array}{l}\text { Do you have any symptoms } \\
\text { such as fever, cough, sore } \\
\text { throat, chest tightness, diarrhea } \\
\text { or fatigue? }\end{array}$ & -0.279 & 0.757 & $0.362-1.583$ & 0.459 & 0.116 & 1.124 & $0.386-3.273$ & 0.831 & -1.368 & 0.255 & $0.068-0.959$ & $0.043^{*}$ \\
\hline & $\begin{array}{l}\text { Have you felt any improvement } \\
\text { since admission? }\end{array}$ & 0.225 & 1.253 & $0.818-1.918$ & 0.300 & -0.088 & 0.916 & $0.481-1.743$ & 0.789 & 0.353 & 1.423 & $0.682-2.969$ & 0.348 \\
\hline
\end{tabular}

N, Number; Cl, confidence interval; OR, odds ratio; COVID-19, coronavirus disease 2019. 
TABLE 3 | Gender-influencing factors of depression symptoms in isolated COVID-19 patients.

\begin{tabular}{|c|c|c|c|c|c|c|c|c|c|c|c|c|c|}
\hline \multirow[t]{2}{*}{ Variables } & & \multicolumn{4}{|c|}{ All $(N=216)$} & \multicolumn{4}{|c|}{ Male $(N=124)$} & \multicolumn{4}{|c|}{ Female $(N=92)$} \\
\hline & & $B$ & OR & $95 \% \mathrm{Cl}$ & $P$ & $B$ & OR & $95 \% \mathrm{Cl}$ & $P$ & $B$ & OR & $95 \% \mathrm{Cl}$ & $P$ \\
\hline \multirow[t]{14}{*}{ Depression } & Gender & -0.178 & 0.837 & $0.378-1.855$ & 0.661 & & & & & & & & \\
\hline & Age & -0.032 & 0.968 & $0.919-1.020$ & 0.220 & -0.152 & 0.859 & $0.767-0.962$ & $0.009^{*}$ & 0.023 & 1.023 & $0.939-1.114$ & 0.604 \\
\hline & Marital status & 0.993 & 2.700 & $1.033-7.055$ & $0.043^{*}$ & 3.415 & 30.409 & $3.485-265.350$ & $0.002^{*}$ & -0.225 & 0.798 & $0.175-3.636$ & 0.771 \\
\hline & Educational level & -0.011 & 0.989 & $0.536-1.825$ & 0.972 & 0.813 & 2.256 & $0.734-6.934$ & 0.156 & -0.474 & 0.623 & $0.236-1.641$ & 0.338 \\
\hline & Occupation & 0.205 & 1.227 & $0.979-1.539$ & 0.075 & 0.391 & 1.479 & $0.985-2.221$ & 0.059 & 0.196 & 1.216 & $0.849-1.742$ & 0.285 \\
\hline & Living area & -18.603 & 0.000 & $0.000-0.000$ & 0.999 & -17.949 & 0.000 & $0.000-0.000$ & 0.999 & -19.731 & 0.000 & $0.000-0.000$ & 0.999 \\
\hline & Monthly Family income & -0.228 & 0.797 & $0.483-1.313$ & 0.373 & -0.973 & 0.378 & $0.165-0.867$ & $0.022^{*}$ & 0.511 & 1.667 & $0.701-3.966$ & 0.248 \\
\hline & Hospitalization time & 0.046 & 1.047 & $0.948-1.156$ & 0.368 & 0.226 & 1.253 & $0.992-1.583$ & 0.058 & -0.023 & 0.978 & $0.812-1.177$ & 0.812 \\
\hline & $\begin{array}{l}\text { Are your family members } \\
\text { infected with COVID-19? }\end{array}$ & 1.088 & 2.969 & $1.243-7.095$ & $0.014^{*}$ & 2.991 & 19.903 & $2.616-151.430$ & $0.004^{*}$ & 0.419 & 1.520 & $0.434-5.319$ & 0.513 \\
\hline & $\begin{array}{l}\text { Are your friends infected with } \\
\text { COVID-19? }\end{array}$ & 0.732 & 2.080 & $0.556-7.782$ & 0.277 & 1.154 & 3.170 & $0.228-44.027$ & 0.390 & 0.463 & 1.589 & $0.228-11.076$ & 0.640 \\
\hline & $\begin{array}{l}\text { Are your colleagues infected } \\
\text { with COVID-19? }\end{array}$ & 0.932 & 2.539 & $0.890-7.244$ & 0.081 & 3.075 & 21.639 & $2.571-182.102$ & $0.005^{*}$ & -0.661 & 0.516 & $0.092-2.903$ & 0.453 \\
\hline & $\begin{array}{l}\text { Are your neighbors infected } \\
\text { with COVID-19? }\end{array}$ & -1.515 & 0.220 & $0.043-1.135$ & 0.070 & -26.017 & 0.000 & $0.000-0.000$ & 0.998 & 0.341 & 1.406 & $0.197-10.058$ & 0.734 \\
\hline & $\begin{array}{l}\text { Do you have any symptoms } \\
\text { such as fever, cough, sore } \\
\text { throat, chest tightness, diarrhea } \\
\text { or fatigue? }\end{array}$ & -0.528 & 0.590 & $0.263-1.319$ & 0.198 & -0.771 & 0.463 & $0.126-1.706$ & 0.247 & -1.181 & 0.307 & $0.069-1.366$ & 0.121 \\
\hline & $\begin{array}{l}\text { Have you felt any improvement } \\
\text { since admission? }\end{array}$ & 0.133 & 1.142 & $0.710-1.838$ & 0.584 & 0.263 & 1.301 & $0.570-2.969$ & 0.532 & 0.470 & 1.600 & $0.660-3.875$ & 0.298 \\
\hline
\end{tabular}

N, Number; Cl, confidence interval; OR, odds ratio; COVID-19, coronavirus disease 2019. 
TABLE 4 | Gender-nursing needs of isolated COVID-19 patients ( $N=216)$.

\begin{tabular}{|c|c|c|c|c|c|c|c|c|c|c|c|c|c|}
\hline \multirow[t]{3}{*}{ Variables } & \multicolumn{6}{|c|}{ Male } & \multicolumn{6}{|c|}{ Female } & \multirow[t]{3}{*}{$x^{2}$} \\
\hline & \multicolumn{2}{|c|}{ Need } & \multicolumn{2}{|c|}{ Does not matter } & \multicolumn{2}{|c|}{ Do not need } & \multicolumn{2}{|c|}{ Need } & \multicolumn{2}{|c|}{ Does not matter } & \multicolumn{2}{|c|}{ Do not need } & \\
\hline & $n$ & $\%$ & $n$ & $\%$ & $n$ & $\%$ & $n$ & $\%$ & $n$ & $\%$ & $n$ & $\%$ & \\
\hline $\begin{array}{l}\text { Be understood and } \\
\text { sympathized. }\end{array}$ & 70 & 56.45 & 18 & 14.52 & 36 & 29.03 & 44 & 47.83 & 14 & 15.22 & 34 & 36.95 & 0.410 \\
\hline $\begin{array}{l}\text { Being taken care of by } \\
\text { family members. }\end{array}$ & 33 & 26.61 & 17 & 13.71 & 74 & 59.68 & 33 & 35.87 & 4 & 4.35 & 55 & 59.78 & $0.044^{*}$ \\
\hline Provide for oneself life. & 40 & 32.26 & 15 & 12.10 & 69 & 55.64 & 33 & 35.87 & 10 & 10.87 & 49 & 53.26 & 0.849 \\
\hline $\begin{array}{l}\text { Keep in touch with } \\
\text { colleagues or friends. }\end{array}$ & 81 & 65.32 & 25 & 20.16 & 18 & 14.52 & 59 & 64.13 & 21 & 22.83 & 12 & 13.04 & 0.874 \\
\hline $\begin{array}{l}\text { Get the attention of the } \\
\text { society. }\end{array}$ & 63 & 50.81 & 29 & 23.39 & 32 & 25.80 & 50 & 54.35 & 13 & 14.13 & 29 & 31.52 & 0.216 \\
\hline $\begin{array}{l}\text { You will receive a warm } \\
\text { reception during the } \\
\text { treatment. }\end{array}$ & 103 & 83.06 & 14 & 11.29 & 7 & 5.65 & 78 & 84.78 & 12 & 13.04 & 2 & 2.18 & 0.432 \\
\hline $\begin{array}{l}\text { The treatment } \\
\text { environment is safe and } \\
\text { comfortable. }\end{array}$ & 104 & 83.87 & 15 & 12.10 & 5 & 4.03 & 87 & 94.57 & 5 & 5.43 & 0 & 0 & $0.012^{*}$ \\
\hline $\begin{array}{l}\text { Timely examination and } \\
\text { treatment. }\end{array}$ & 118 & 95.16 & 5 & 4.03 & 1 & 0.81 & 90 & 97.82 & 1 & 1.09 & 1 & 1.09 & 0.420 \\
\hline $\begin{array}{l}\text { Communicate with your } \\
\text { doctor or nurse. }\end{array}$ & 116 & 93.55 & 8 & 6.45 & 0 & 0 & 90 & 97.82 & 1 & 1.09 & 1 & 1.09 & $0.047^{\star}$ \\
\hline $\begin{array}{l}\text { When you are in } \\
\text { hospital, you can confide } \\
\text { in others if you have } \\
\text { unpleasant things. }\end{array}$ & 91 & 73.39 & 25 & 20.16 & 8 & 6.45 & 75 & 81.53 & 12 & 13.04 & 5 & 5.43 & 0.349 \\
\hline $\begin{array}{l}\text { Communicate and talk } \\
\text { with fellow patients. }\end{array}$ & 91 & 73.39 & 24 & 19.35 & 9 & 7.26 & 73 & 79.35 & 15 & 16.30 & 4 & 4.35 & 0.532 \\
\hline $\begin{array}{l}\text { Doctor or nurse can tell } \\
\text { the truth about a } \\
\text { patient's condition. }\end{array}$ & 115 & 92.74 & 8 & 6.45 & 1 & 0.81 & 87 & 94.56 & 4 & 4.35 & 1 & 1.09 & 0.785 \\
\hline $\begin{array}{l}\text { Learn about the spread } \\
\text { of COVID-19. }\end{array}$ & 112 & 90.32 & 11 & 8.87 & 1 & 0.81 & 86 & 93.48 & 6 & 6.52 & 0 & 0 & 0.557 \\
\hline $\begin{array}{l}\text { Understand the effects } \\
\text { and side effects of } \\
\text { therapeutic agents. }\end{array}$ & 121 & 97.58 & 3 & 2.42 & 0 & 0 & 89 & 96.74 & 3 & 3.26 & 0 & 0 & 0.510 \\
\hline $\begin{array}{l}\text { Understand the } \\
\text { prognosis of the disease }\end{array}$ & 120 & 96.77 & 4 & 3.23 & 0 & 0 & 91 & 98.91 & 1 & 1.09 & 0 & 0 & 0.291 \\
\hline $\begin{array}{l}\text { Guidance for preventing } \\
\text { recurrence. }\end{array}$ & 119 & 95.97 & 5 & 4.03 & 0 & 0 & 90 & 97.82 & 2 & 2.18 & 0 & 0 & 0.362 \\
\hline $\begin{array}{l}\text { Understand hospital and } \\
\text { medical team structure. }\end{array}$ & 93 & 75.00 & 22 & 17.74 & 9 & 7.26 & 66 & 71.74 & 21 & 22.83 & 5 & 5.43 & 0.597 \\
\hline $\begin{array}{l}\text { Know the names of } \\
\text { doctors and nurses. }\end{array}$ & 79 & 63.71 & 34 & 27.42 & 11 & 8.87 & 59 & 64.13 & 27 & 29.35 & 6 & 6.52 & 0.802 \\
\hline
\end{tabular}

${ }^{*} P<0.05$.

that the workplace of participants may be in the outbreak area, which could company bankruptcy or patient unemployment. Male participants as pillars of their family, are particularly more likely to experience anxiety symptoms due to these stresses (Shi et al., 2020). On the other hand, occupation and COVID-19 accompanying symptoms can affect the anxiety symptoms in female participants. Female participants who were unemployed or freelance workers and who developed symptoms related to COVID-19 had higher anxiety. This may be related to the influence of job instability on their economic conditions (Xiao et al., 2020) and/or physical discomfort caused by the disease (Xue et al., 2008). More specifically, previous studies have shown that hypoxia and dyspnea are among the most common symptoms for COVID-19 patients (Chen N. et al., 2020). In this context, dyspnea is significantly and positively correlated with negative emotions such as anxiety, depression, and fear (Jiao, 2013).

Previous studies on depression in COVID-19 patients have found that those who are married or have other family members with the disease are more likely to develop depression symptoms. In particular, married men aged 18-39 years with lower family incomes and family members or colleagues with COVID-19 are more likely to suffer from depression (Shi et al., 2020; Xiao et al., 2020). In this regard, individuals with those attributes are also more likely undertake heavy work tasks and family support roles, thus incurring greater psychological burdens and negative emotions (Xiao et al., 2020). Male isolated COVID-19 patients with lower monthly household incomes may experience even more depressive symptoms due to financial stress (Cheng et al., 2020), while 
those with infected family members and colleagues may develop additional concerns. In general, disease-related uncertainties may lead to depression during isolation, particularly when there is otherwise a high amount of close daily contact with family members and/or colleagues. In other words, the isolation environment may create a lack of information, thus increasing the level of concern.

The influencing factors for anxiety and depression differ between genders. This may be related to the different roles, responsibilities, and jobs held by men and women in society (Weich et al., 1998). Further, research has shown that women are more prone to negative emotions when facing stressful events, particularly when under the influences of physiological and cognitive factors (McLean and Hope, 2010). When treating COVID-19 patients, medical staff should therefore implement targeted psychological interventions aimed at the different psychological characteristics of male and female patients. This will provide a better way to target the unique elements that contribute to anxiety and depression while enhancing confidence and courage among those facing the disease.

\section{Analyzing Nursing Needs}

In this study, more than $95 \%$ of patients reported on timely examinations and treatments, an understanding of the disease prognosis, understanding the effects and side effects of therapeutic agents, and guidance for preventing recurrence. This may be related to the nature and severity of COVID-19 itself. Indeed, novel coronaviruses may pose serious harm to humans, and are typically highly contagious (Wei and Li, 2020). For COVID-19, the number of deaths in China now exceeds the total number during the SARS epidemic of 2002-2003 (Jingwei Network, 2003), and there is no specific drug that targets the virus. Patients may therefore express more nursing needs related to examinations, drug treatments, prognoses, and recurrence and prevention guidance due to increased worries and uncertainties about their conditions and outlooks.

Notably, female participants reported greater need than male participants in three specific areas, including being cared for by family members, a safe and comfortable treatment environment, and communication with doctors and nurses. These differences were statistically significant, which is consistent with previous research ( $\mathrm{Li}$ et al., 2003). In general, this may be due to the fact that female patients are more sensitive to the perception of negative emotions and more likely to have physiological/psychological reactions related to negative emotions than males (McLean and Anderson, 2009; Zhang and Li, 2020). Healthcare workers should therefore pay increased attention to the unique psychological needs of patients while increasing the overall level of communication. This includes a particular focus on providing timely diagnosis, treatment, and nursing information to female patients.

\section{LIMITATIONS}

This study had two main limitations. First, it only investigated COVID-19 patients at one makeshift hospital in Wuhan, which may have resulted in selection bias. Second, only general data from patients were analyzed. In this case, the effects of personality traits, disease cognition, social support, and other factors are unknown. Future studies should address both these issues, thus contributing to a more comprehensive discussion on the psychological status of COVID-19 patients.

\section{CONCLUSION}

Of the isolated COVID-19 patients included in this analysis, $21.76 \%$ had anxiety symptoms, while $17.59 \%$ had depression symptoms. When broken down to look at gender, there were different influencing factors for both anxiety and depression. More specifically, female patients reported greater psychological nursing needs than male patients. While treating the illness, healthcare workers should therefore pay increased attention to any emotional changes, especially among patients who are more susceptible to anxiety and depression. Targeted nursing should thus be implemented to meet specific nursing needs, while psychological interventions should aim to promote overall mental health while preventing the development of more serious mental diseases.

\section{DATA AVAILABILITY STATEMENT}

The raw data supporting the conclusions of this article will be made available by the authors, without undue reservation.

\section{ETHICS STATEMENT}

The studies involving human participants were reviewed and approved by the Xiang Ya Nursing School of Central South University (Ethical Grant Number: E201946). - Written informed consent to participate in this study was provided by the patient/participants.

\section{AUTHOR CONTRIBUTIONS}

JPZ was the primary investigator of the study and provided comments and ideas, and revised this manuscript. YL conducted the data analysis and contributed to writing the manuscript. JL and JZ helped with the data analysis and coding and contributed to writing the manuscript. LD and FW helped with the questionnaire survey. ZY contributed conceptually to data generation and analysis and suggested revisions. All authors contributed to the article and approved the submitted version.

\section{ACKNOWLEDGMENTS}

Thanks to all the participants and researchers involved in this study. 


\section{REFERENCES}

Amirian, E. S. (2020). Potential fecal transmission of SARS-CoV-2: current evidence and implications for public health. Int. J. Infec. Dis. 95, 363-370. doi: 10.1016/j.ijid.2020.04.057

Arab-Mazar, Z., Sah, R., Rabaan, A. A., Dhama, K., and Rodriguez-Morales, A. J. (2020). Mapping the incidence of the COVID-19 hotspot in Iran - implications for travellers. Travel Med. Infect. Dis. 34:101630. doi: 10.1016/j.tmaid.2020. 101630

Chen, N., Zhou, M., Dong, X., Qu, J., Gong, F., Han, Y., et al. (2020). Epidemiological and clinical characteristics of 99 cases of 2019 novel coronavirus pneumonia in Wuhan, China: a descriptive study. Lancet 395, 507-513. doi: 10.1016/S0140-6736(20)30211-7

Chen, Y. H., and Li, W. (2020). The Clinical Symptoms, Classification and Diagnosis of COVID-19. Genomics Appl. Biol. 39, 3904-3907. doi: 10.13417/ j.gab.039.003904

Chen, Z., Zhuang, Y. J., Li, J., Yang, X. L., Li, J., Feng, Y., et al. (2020). Medical management of hospitals designated for patients with COVID-19. Chin. J. Nosocomiol. 30, 821-825. doi: 10.11816/cn.ni.2020-200237

Cheng, J. G., Tan, X. D., Zhang, L., Zhu, S. R., Yao, H., and Liu, B. (2020). Research on the psychological status and influencing factors of novel coronavirus pneumonia patients and people under medical observation. J. Nurs. Adm. 20, 247-251.

Fan, P., Aloweni, F., Lim, S. H., Ang, S. Y., Perera, K., Quek, A. H., et al. (2020). Needs and concerns of patients in isolation care units - learnings from COVID19: a reflection. World J. Clin. Cases 8, 1763-1766. doi: 10.12998/wjcc.v8.i10. 1763

Fu, Y. N., and Luo, X. (2011). The role of psychosocial factors in the significant emergencies disease responding. Chin. J. Soc. Med. 28, 279-280.

Gu, Y., Zhu, Y., Xu, F., Xi, J., and Xu, G. (2020). Factors associated with mental health outcomes among patients with COVID-19 treated in the Fangcang shelter hospital in China. Asia Pacific Psychiatry 13:e12443. doi: 10.1111/appy. 12443

Jiao, T. (2013). A Prevalence of Anxiety and Depression in Patients With an Acute Exacerbation of COPD. Hefei: Anhui Medical University.

Jingwei Network (2003). The Ministry of Health Announced the Last Daily Outbreak: No SARS Patients in Mainland China. Avaliable online at: http:// www.huaxia.com/xw/dlxw/2003/08/234782.html (accessed August 17, 2003).

Kwek, S. K., Chew, W. M., Ong, K. C., Ng, A. W., Lee, L. S., Kaw, G., et al. (2006). Quality of life and psychological status in survivors of severe acute respiratory syndrome at 3 months postdischarge. J. Psychosom. Res. 60, 513-519. doi: 10. 1016/j.jpsychores.2005.08.020

Li, S. J., Yang, S., Zhang, Y. R., Zhang, Y., Wang, Y. L., Sun, C. L., et al. (2003). Investigation of SARS patients' health service needs. Chin. J. Nurs. 12, 18-20.

McLean, C. P., and Anderson, E. R. (2009). Brave men and timid women? A review of the gender differences in fear and anxiety. Clin. Psychol. Rev. 29, 496-505. doi: 10.1016/j.cpr.2009.05.003

McLean, C. P., and Hope, D. A. (2010). Subjective anxiety and behavioral avoidance: gender, gender role, and perceived confirmability of self-report. J. Anxiety Disord. 24, 494-502. doi: 10.1016/j.janxdis.2010.03.006

National Health Commission of the People's Republic of China (2020). Interpretation of COVID-19 Diagnosis and Treatment Protocol (trial version 7). Avaliable online at: http://www.nhc.gov.cn/yzygj/s7652m/202003/ a31191442e29474b98bfed5579d5af95.shtml (accessed March 4, 2020).

Nie, X. D., Wang, Q., Wang, M. N., Zhao, S., Liu, L., Zhu, Y. L., et al. (2020). Anxiety and depression and its correlates in patients with coronavirus disease 2019 in Wuhan. Int. J. Psychiatry Clin. Pract. 1-6. doi: 10.1080/13651501.2020.1791345. [Epub ahead of print].
Pang, S. X. (2016). Effect of nursing intervention on negative emotion and quality of life of patients with acute respiratory infection. J. Qilu Nurs. 22, 32-34. doi: 10.3969/j.issn.1006-7256.2016.09.017

Sharma, A., Pillai, D. R., Lu, M., Doolan, C., Leal, J., Kim, J., et al. (2020). Impact of isolation precautions on quality of life: a meta-analysis. J. Hosp. Infect. 105, 35-42. doi: 10.1016/j.jhin.2020.02.004

Sheng, Y., Gao, F. L., and Zhu, L. X. (2003). Investigation and analysis for nursing needs of SARS patients with "functional health patterns" of Gordon. Chin. J. Nurs. 12, 4-6.

Shi, L., Lu, Z. A., Que, J. Y., Huang, X. L., Liu, L., Ran, M. S., et al. (2020). Prevalence of and risk factors associated with mental health symptoms among the general population in china during the coronavirus disease 2019 pandemic. JAMA Netw. Open 3:e2014053. doi: 10.1001/jamanetworkopen.2020.14053

Wang, C., Horby, P. W., Hayden, F. G., and Gao, G. F. (2020). A novel coronavirus outbreak of global health concern. Lancet 395, 470-473. doi: 10.1016/S01406736(20)30185-9

Wang, Z. Y., Gao, X. L., Cui, Y. Y., Huang, J. Y., and Qian, H. (2020). Analysis of anxiety and depression in patients with mild coronary pneumonia. Psychologies 15, 40-42. doi: 10.19738/j.cnki.psy.2020.20.014

Wei, Z. X., and Li, Q. F. (2020). An overview of the clinical manifestations of novel coronavirus infection and its laboratory detection methods. Int. J. Lab. Med. 41, 988-992. doi: 10.3969/j.issn.1673-4130.2020.08.023

Weich, S., Sloggett, A., and Lewis, G. (1998). Social roles and gender difference in the prevalence of common mental disorders. Br. J. Psychiatry 173, 489-493. doi: 10.1192/bjp.173.6.489

World Health Organization (WHO) (2021). World Health Organization Coronavirus Disease (COVID-19) Pandemic. Avaliable online at: https://www.who.int/emergencies/diseases/novel-coronavirus-2019 (accessed April 30, 2021).

Xiao, J. L., Chen, Y., Fang, F., Liu, W. T., Zhong, Y. Y., Tao, J., et al. (2020). Public anxiety and depression and its influencing factors under public health emergencies. Modern Prev. Med. 47, 3557-3562.

Xiong, J., Jiang, W. L., Zhou, Q., Hu, X. Q., and Liu, C. Y. (2020). Clinical characteristics, treatment, and prognosis in 89 cases of COVID-19. Med. J. Wuhan Univ. 41, 542-546. doi: 10.14188/j.1671-8852.2020.0103

Xue, Y. Z., Lu, L., Liang, Z. Q., Xu, Y., and Zhang, K. R. (2008). Investigation on psychosomatic symptoms of SARS patients at different stages. Chin. Prev. Med. $04,268-270$.

Yang, H. (2004). Exploration of Response of Psychology and Psychological Nursing Intervention in SARS Patients. Jinzhong: Shanxi Medical University.

Zhang, Y. (2012). Mental health needs survey and intervention analysis of infectious disease patients. Med. Innov. China 9, 72-73. doi: 10.3969/j.issn. 1674-4985.2012.24.041

Zhang, Y. Z., and Li, X. F. (2020). Investigation and analysis of current situation of pain management in inpatients. Clin. Res. Pract. 5, 3-5. doi: 10.19347/j.cnki. 2096-1413.202010002

Zigmond, A. S., and Snaith, R. P. (1983). The hospital anxiety and depression scale. Acta Psych. Scand 67, 361-370. doi: 10.1111/j.1600-0447.1983.tb09716.x

Conflict of Interest: The authors declare that the research was conducted in the absence of any commercial or financial relationships that could be construed as a potential conflict of interest.

Copyright (c) $2021 \mathrm{Li}$, Li, Yang, Zhang, Dong, Wang and Zhang. This is an open-access article distributed under the terms of the Creative Commons Attribution License (CC BY). The use, distribution or reproduction in other forums is permitted, provided the original author(s) and the copyright owner(s) are credited and that the original publication in this journal is cited, in accordance with accepted academic practice. No use, distribution or reproduction is permitted which does not comply with these terms. 